\title{
Analysing the Hipparcos epoch photometry of $\lambda$ Bootis stars
}

\author{
E. Paunzen, P. Reegen
}

Institut für Astronomie, Türkenschanzstrasse 17, 1180 Vienna, Austria

\begin{abstract}
We performed a homogeneous analysis of the available Hipparcos epoch photometry for all members of the $\lambda$ Bootis group. Besides the known $\delta$ Scuti like pulsation frequencies, we searched for eclipses due to binarity or rotational induced variability. The already known frequencies of HD 15165 are confirmed with an additional lower single frequency, which might be caused by an accretion episode. But we are not able to rule out a possible aliasing due to the temporal sampling of the data set. Furthermore, three candidates (HD 15759, HD 120500, and HD 149130) for a possible eclipse were detected. Otherwise, the frequency analysis reveals a null result within our strict detection limits.

Individual Objects: HD 319, HD 6870, HD 7908, HD 11413, HD 13755, HD 15165, HD 15759, HD 23392, HD 24472, HD 30422, HD 31295, HD 35242, HD 64491, HD 74873, HD 75654, HD 81290, HD 83041, HD 83277, HD 84123, HD 84948, HD 87271, HD 91130, HD 101108, HD 102541, HD 105058, HD 106223, HD 107233, HD 110411, HD 111005, HD 111604, HD 111786, HD 120500, HD 120896, HD 125162, HD 130767, HD 141851, HD 142703, HD 149130, HD 153747, HD 154153, HD 156954, HD 160928, HD 168740, HD 170680, HD 171948, HD 175445, HD 183324, HD 192640, HD 198160, HD 204041, HD 210111, HD 221756
\end{abstract}

\section{Introduction}

In this paper, we present a homogeneous analysis of the Hipparcos epoch photometry (Perryman et al. 1997) for members of the $\lambda$ Bootis group. This small group comprises late B- to early F-type, Population I stars which are metal weak (particularly the Fe group elements), but with the clear exception of $\mathrm{C}, \mathrm{N}, \mathrm{O}$, and $\mathrm{S}$. Only a maximum of about $2 \%$ of all objects in the relevant spectral domain are believed to be $\lambda$ Bootis type stars. See Paunzen (2004) for a review of the astrophysical details of this group.

An extensive survey to analyse the pulsational characteristics of the $\lambda$ Bootis stars has been presented by Paunzen et al. (2002). They conclude that at least $70 \%$ of all $\lambda$ Bootis types stars ( $=33$ objects) inside the classical instability strip pulsate with 


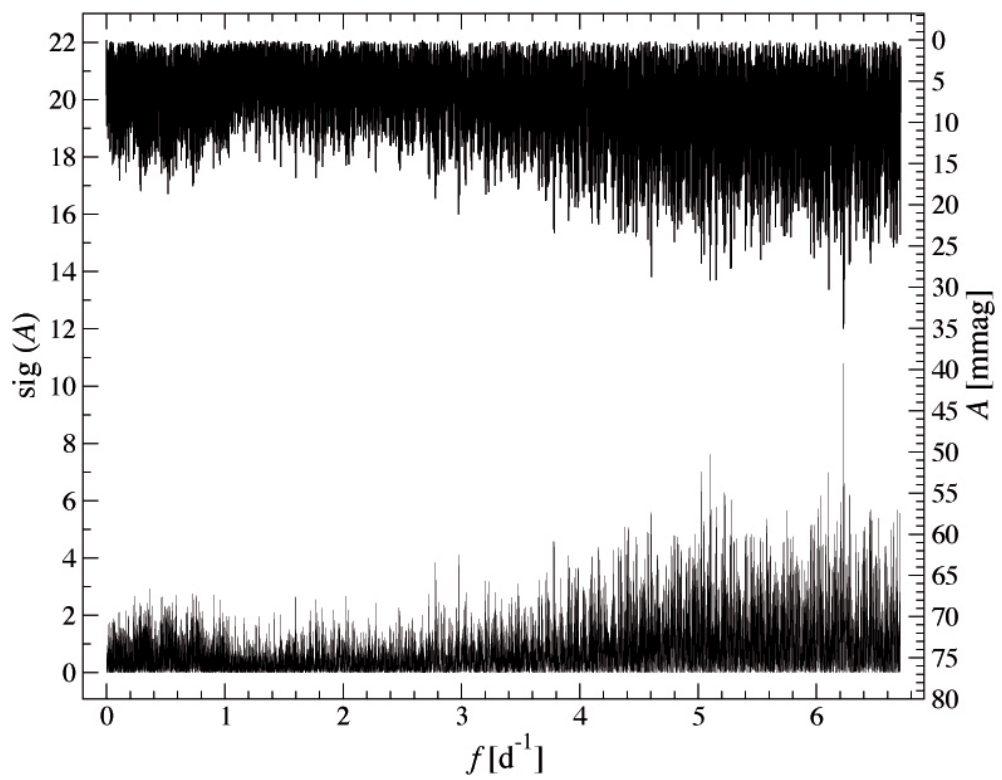

Figure 1: Significance and amplitude (inverted axis) spectrum of HD 15165.

frequencies typical for $\delta$ Scuti type pulsators. The amplitudes do, in general, not exceed a few mmags.

For this analysis we selected all bona-fide $\lambda$ Bootis stars, 52 objects in total, which are included in the Hipparcos epoch photometry catalogue. It is known that these data sets are not optimally distributed to detect new $\delta$ Scuti type pulsators (Koen 2001).

The aim of this project is to find significant frequencies in the photometric data sets, especially in a domain other than the classical $\delta$ Scuti one (e.g., eclipses due to binarity or rotational induced variability).

\section{Fourier Analysis}

The 52 light curves were analyzed using SigSpEC (Reegen 2007), a technique that provides a clean statistical treatment of DFT spectra. It assigns a spectral significance (hereafter abbreviated by 'sig') to a DFT amplitude, also taking into account the statistical behaviour of white noise in Fourier space depending not only on amplitude, but also on frequency and phase (and implicitly on the time-domain sampling).

Table 1 contains the numbers of data points, the time bases, and the Nyquist frequencies (determined by the median of time step widths between consecutive data points) for the 52 stars. The last three columns refer to the frequency, sig, and amplitude of the most significant signal component identified in the DFT spectrum. Setting the threshold for the reliability of a peak to $\operatorname{sig}=5$, we consider peaks significant, if the False-Alarm Probability of white noise to generate such a Fourier amplitude is below 0.00001 . 
Table 1: HD identifiers, numbers of data points, time bases, Nyquist frequencies, and frequencies, sigs, and amplitudes of the dominant signal for 52 HIPPARCOS stars.

\begin{tabular}{|c|c|c|c|c|c|c|}
\hline HD & $\#$ & $\Delta t[\mathrm{~d}]$ & $f_{\mathrm{Ny}}\left[\mathrm{d}^{-1}\right]$ & $f\left[\mathrm{~d}^{-1}\right]$ & $\max (\mathrm{sig})$ & $A[\mathrm{mmag}]$ \\
\hline 319 & 124 & 1140.56 & 6.70780 & 0.22178 & 2.079 & 16.457 \\
\hline 6870 & 159 & 1183.23 & 6.70601 & 2.07181 & 4.313 & 5.892 \\
\hline 7908 & 143 & 1132.14 & 6.70693 & 4.54884 & 3.418 & 10.929 \\
\hline 11413 & 129 & 1187.85 & 6.70603 & 1.56827 & 3.305 & 6.170 \\
\hline 13755 & 160 & 1190.04 & 6.70601 & 5.52756 & 3.384 & 21.952 \\
\hline 15165 & 75 & 887.28 & 6.70959 & 6.22812 & 10.801 & 34.862 \\
\hline 15759 & 90 & 1165.52 & 6.70601 & 0.34642 & 1.713 & 221.714 \\
\hline 23392 & 74 & 1076.89 & 6.70780 & 1.47128 & 3.516 & 11.379 \\
\hline 24472 & 150 & 1159.45 & 6.70602 & 1.11333 & 4.400 & 6.603 \\
\hline 30422 & 174 & 1156.75 & 6.70690 & 1.89886 & 4.004 & 3.779 \\
\hline 31295 & 94 & 918.38 & 6.70783 & 0.16681 & 2.598 & 15.696 \\
\hline 35242 & 77 & 919.19 & 6.70779 & 4.48962 & 2.120 & 3.830 \\
\hline 64491 & 77 & 1091.75 & 6.70869 & 0.17311 & 4.441 & 8.302 \\
\hline 74873 & 89 & 964.64 & 6.70869 & 0.74176 & 3.108 & 4.251 \\
\hline 75654 & 162 & 1182.55 & 6.70689 & 2.30658 & 4.696 & 4.959 \\
\hline 81290 & 126 & 1155.28 & 6.70510 & 2.41323 & 1.867 & 20.599 \\
\hline 83041 & 159 & 1113.46 & 6.70691 & 3.02860 & 4.328 & 9.897 \\
\hline 83277 & 121 & 1113.55 & 6.70781 & 1.94421 & 4.707 & 9.762 \\
\hline 84123 & 139 & 1093.91 & 6.70692 & 0.20711 & 4.711 & 7.591 \\
\hline 84948 & 153 & 1183.07 & 6.70602 & 2.04655 & 2.707 & 12.374 \\
\hline 87271 & 83 & 1083.33 & 6.70689 & 0.44663 & 3.492 & 14.165 \\
\hline 91130 & 123 & 1083.48 & 6.70782 & 2.42232 & 2.406 & 9.078 \\
\hline 101108 & 125 & 1058.26 & 6.70689 & 0.95198 & 2.815 & 14.922 \\
\hline 102541 & 126 & 1102.90 & 6.70602 & 5.16591 & 4.432 & 10.537 \\
\hline 105058 & 160 & 1186.58 & 6.70602 & 4.77779 & 3.958 & 14.834 \\
\hline 106223 & 92 & 1141.19 & 6.70690 & 0.47121 & 3.426 & 10.708 \\
\hline 107233 & 206 & 1171.91 & 6.70690 & 2.00583 & 3.794 & 10.254 \\
\hline 110411 & 67 & 1134.16 & 6.70689 & 5.26716 & 1.753 & 17.218 \\
\hline 111005 & 81 & 1166.32 & 6.70690 & 1.75893 & 2.806 & 23.616 \\
\hline 111604 & 96 & 1145.01 & 6.70601 & 5.33004 & 3.989 & 10.206 \\
\hline 111786 & 118 & 1146.62 & 6.70599 & 0.85562 & 2.530 & 12.755 \\
\hline 120500 & 104 & 1166.31 & 6.70692 & 0.00009 & 3.753 & 1492.025 \\
\hline 120896 & 84 & 1164.90 & 6.70691 & 0.15475 & 3.751 & 17.126 \\
\hline 125162 & 148 & 1151.21 & 6.70693 & 0.18339 & 2.406 & 10.840 \\
\hline 130767 & 106 & 1139.14 & 6.70511 & 6.42451 & 3.779 & 5.338 \\
\hline 141851 & 105 & 1085.31 & 6.70689 & 3.94765 & 3.006 & 3.287 \\
\hline 142703 & 96 & 1122.67 & 6.70691 & 0.50608 & 2.523 & 9.123 \\
\hline 149130 & 125 & 1123.63 & 6.70689 & 0.66142 & 2.152 & 57.445 \\
\hline 153747 & 101 & 1121.34 & 6.70779 & 6.33698 & 2.984 & 8.363 \\
\hline 154153 & 80 & 1121.68 & 6.70602 & 6.56142 & 1.971 & 3.130 \\
\hline 156954 & 73 & 916.68 & 6.70693 & 0.23387 & 3.502 & 23.215 \\
\hline 160928 & 64 & 782.99 & 6.70778 & 2.46921 & 3.929 & 5.590 \\
\hline 168740 & 118 & 1122.39 & 6.70692 & 0.92413 & 2.743 & 9.432 \\
\hline 170680 & 65 & 1097.79 & 6.70781 & 1.93363 & 4.095 & 3.556 \\
\hline 171948 & 248 & 1032.01 & 6.70689 & 2.32768 & 4.375 & 4.204 \\
\hline 175445 & 113 & 1094.12 & 6.70693 & 0.20979 & 3.306 & 5.174 \\
\hline 183324 & 98 & 1089.25 & 6.70601 & 0.37489 & 3.395 & 6.224 \\
\hline 192640 & 143 & 1153.57 & 6.70601 & 2.28322 & 2.569 & 4.354 \\
\hline 198160 & 144 & 1093.73 & 6.70603 & 3.25459 & 1.721 & 6.560 \\
\hline 204041 & 63 & 953.84 & 6.70688 & 0.14368 & 2.755 & 15.575 \\
\hline 210111 & 76 & 1097.44 & 6.70600 & 2.36706 & 3.122 & 4.643 \\
\hline 221756 & 118 & 1152.20 & 6.70510 & 0.80059 & 2.442 & 7.570 \\
\hline
\end{tabular}


Table 2: Significant signal components for HD 15165. The right panel lists the corresponding frequencies and amplitudes (in a narrow band system) from Liu et al. (1996).

\begin{tabular}{rrr|rr}
\hline$f\left[\mathrm{~d}^{-1}\right]$ & $\max (\mathrm{sig})$ & $A[\mathrm{mmag}]$ & $f\left[\mathrm{~d}^{-1}\right]$ & $A[\mathrm{mmag}]$ \\
\hline 6.228116 & 10.8 & 33.486 & 6.2273 & 20.08 \\
6.512936 & 5.6 & 16.047 & 6.5186 & 11.02 \\
0.761334 & 5.8 & 11.948 & & \\
\hline
\end{tabular}

The only object satisfying this requirement is HD 15165 with a peak sig of 10.8 at a frequency of $6.22 \mathrm{~d}^{-1}$. Its DFT amplitude spectrum (also containing sig) is displayed in Figure 1, and a list of the three significant signal components identified by SIGSPEC through a combination of consecutive prewhitening and least-squares fitting is provided in Table 2 .

\section{Results and Conclusions}

We only found significant frequencies for HD 15165 (VW Arietis), which was the target of the fifth STEPHI campaign (Liu et al. 1996). They have detected seven significant frequencies between 6.23 and $12.85 \mathrm{~d}^{-1}$. Two of them match our results within the given errors very well (Table 2). The lower frequency was not detected by Liu et al. (1996), which might be due to their reduction algorithm that automatically corrects the light curves for zero points from night to night. If we speculate that this frequency is due to a rotational coupled phenomenon, it might point toward an accretion episode (Kamp \& Paunzen 2002). Such a variability was also found in other stars with shells and accretion disks (Sudzius \& Sperauskas 1996). On the other hand, we are not able to rule out a possible aliasing due to the temporal sampling of the data set. Further observations are needed to prove the existence of this frequency.

If we compare our results with those of the already known pulsating $\lambda$ Bootis stars, we find that the Nyquist frequencies of the Hipparcos epoch photometry is always below the frequencies listed in Paunzen et al. (2002; Table 4). Our null result is therefore fully consistent with their data sets.

The binarity among the $\lambda$ Bootis group is still a controversial topic (Stütz \& Paunzen 2007). Although there are several spectroscopic binaries known to be members of the group, no eclipsing binaries have been detected so far. A possible eclipse would yield a large amplitude, but a small $\sigma$ in our analysis due to the temporal data distribution. Table 1 includes three promising candidates: HD 15759, HD 120500 and HD 149130. A closer inspection of the individual data sets reveals that exactly one measurement for each star causes these large amplitudes. However, they will be targets of further investigations. 
Acknowledgments. This work was supported by the Fonds zur Förderung der wissenschaftlichen Forschung (FWF, projects P14546-PHY and P14984-PHY).

\section{References}

Kamp, I., \& Paunzen, E. 2002, MNRAS 335, L45

Koen, C. 2001, MNRAS 321, 44

Liu, Y. Y., Jiang, S. Y., Michel, E., et al. 1996, A\&AS 120, 179

Paunzen, E. 2004, The A-Star Puzzle, held in Poprad, Slovakia, July 8-13, 2004.

Edited by J. Zverko, J. Ziznovsky, S. J. Adelman, and W. W. Weiss, IAU

Symposium, No. 224. Cambridge, UK: Cambridge University Press, 2004., p. 443

Paunzen, E., Handler, G., Weiss, W. W., et al. 2002, A\&A 392, 515

Perryman, M. A. C., \& the Hipparcos Science Team 1997, The Hipparcos and Tycho Catalogues (ESA SP-1200; Noordwijk: ESA)

Reegen, P. 2007, A\&A 467, 1353

Stütz, C., \& Paunzen, E. 2007, A\&A 458, L17

Sudzius, J., \& Sperauskas, J. 1996, AN 317, 349 